\title{
EL DERECHO A UN MEDIO AMBIENTE SANO Y LA ENCRUCIJADA DE LOS ALIMENTOS TRANSGÉNICOS ${ }^{1}$ The Right to a Healthy Environment and the Crossroads \\ of Transgenic Foods
}

\author{
Agustín Viguri Perea² \\ Maria Chiara Marullo ${ }^{3}$
}

Fecha de Recepción: 4 de noviembre de 2015

Fecha de Aceptación: 2 de diciembre de 2015

SUMARIO: 1. Introducción; 2. Conexión existente entre el Derecho a un Ambiente sano y las

actividades de las Empresa; 3. La encrucijada de los alimentos transgénicos: Estados Unidos,

Europa y la OMC; 4. Conclusiones; 5. Referencias bibliográficas.

\footnotetext{
${ }^{1}$ El presente trabajo se ha realizado bajo la acción prevista por la Dirección General de Justicia de la Comisión Europea (JUST/2013/ ACTION GRANTS)

${ }^{2}$ Dr. Agustín Viguri Perea, TU (Universidad Jaume I -UJI- de Castellón de la Plana) Licenciado en Derecho norteamericano (University of Pittsburgh, USA) Correo: viguri@uji.es.

${ }^{3}$ Dra. Maria Chiara Marullo, investigadora del proyecto: BUSINESS \& HUMAN RIGHTS CHALLENGES FOR CROSS BORDER LITIGATION IN THE EUROPEAN UNION. ACTION GRANT OF THE EU. Correo: marullo@uji.es
} 


\section{COMO SE CITA ESTE ARTÍCULO (APA 6)}

Viguri Perea, Agustín; Marullo, Maria Chiara (2016). El derecho a un medio ambiente sano y la encrucijada de los alimentos transgénicos. Revista Jurídica Mario Alario D’Filippo, VIII (15), pág 100-111.

\section{RESUMEN}

Este trabajo aborda los problemas relacionados con el derecho ambiental, los derechos humanos, el impacto de ambos en los procesos de las Compañías Multinacionales y el derecho a la Salud desde el punto de vista del derecho comparado. Al mismo tiempo, se trata esta cuestión desde una perspectiva acorde al derecho de casos. Se parte de la idea de que uno de los sectores financieros de mayor actualidad en el momento presente a nivel mundial es el relativo a los alimentos transgénicos.

\section{PALABRAS CLAVES}

Derecho ambiental, derechos humanos, compañías transnacionales, método del caso, derecho a la salud, el dilema de los alimentos transgénicos.

\section{ABSTRACT}

This paper faces the problems related to Environmental Law, Human Rights, the impact of both in the processes of Multinational Corporations, and the right to health all together from a comparative point of view. At the same time it deals with this topic from a case law perspective. The basic idea in the beginning is that one of the financial sectors which is in the limelight all over the world in the present moment is precisely the subject concerning genetically modified foods.

\section{KEYWORDS}

Human Rights, Environmental Law, Multinational Corporations, Case Law, Right to Health, the dilemma of genetically modified foods. 


\section{INTRODUCCIÓN}

El 15 de marzo de 2016 la Organización Mundial de la Salud (OMS) en su informe "Ambientes saludables y prevención de enfermedades: Hacia una estimación de la carga de morbilidad atribuible al medio ambiente" ${ }^{4}$ hizo referencia a las estadísticas del año 2012 que reflejan que 12,6 millones de personas, una cuarta parte del total mundial de muertes, perdieron la vida por vivir o trabajar en ambientes poco saludables ${ }^{5}$. Los factores de riesgo ambiental como la contaminación del aire, el agua y el suelo ${ }^{6}$ la exposición a los productos químicos, el cambio climático y la radiación ultravioleta contribuyen a más de 100 enfermedades o traumatismos. Las nuevas estadísticas muestran un panorama desolador donde las muertes por enfermedades que pueden atribuirse a la contaminación del aire han aumentado hasta la cifra de 8,2 millones. Sobre este aspecto se muestran mapas en los que se evidencia como más del $92 \%$ de la población que habita en zonas urbanas ha estado expuesta a niveles de calidad de aire que exceden los límites recomendados por la $\mathrm{OMS}^{7}$ En Europa la contaminación ha sido la causa de los fallecimientos prematuros de más de 430.000 personas en 2011, según indican datos procedentes de la Agencia Europea del Medioambiente ${ }^{8}$, mientras que los accidentes cerebrovasculares, los cánceres y las neumopatías crónicas constituyen actualmente casi dos terceras partes del total de muertes debidas la insalubridad del medio ambiente. Ello se debe en una buena medida a que, a pesar de la existencia de normas estrictas,

${ }^{4}$ El informe se puede consultar en su versión inglesa: Prüss-Ustün, A, et. al (2016) Preventing disease through healthy environments: A global assessment of the burden of disease from environmental risks. World Health Organization. Recuperado de: http://apps.who. int/iris/bitstream/10665/204585/1/9789241565196_eng.pdf?ua=1.

${ }^{5}$ Más información en: Organización Mundial de la Salud. (2016) Cada año mueren 12,6 millones de personas a causa de la insalubridad del medio ambiente. Comunicado de prensa. Recuperado de: http://www.who.int/mediacentre/news/releases/2016/deathsattributable-to-unhealthy-environments/es/

${ }^{6}$ Con respecto a la contaminación del aire: El 28 de septiembre de 2016 la BBC publicó un mapa que muestra la gran contaminación del aire que respiramos, más información en: http://www.bbc.com/mundo/noticias-37501943 Véase también: Organización Mundial de la Salud (2016) Health and sustainable development. Recuperado de: http://www.who.int/sustainable-development/ news-events/breath-life/en/ ; The World Bank (2016) The cost of air pollution : strengthening the economic case for action (English) Recuperado de: http://documents.worldbank.org/curated/en/781521473177013155/The-cost-of-air-pollution-strengthening-theeconomic-case-for-action; Frisch, W (2015) Derecho penal y protección del clima: Posibilidades y límites de una contribución del Derecho penal a la protección del clima conforme a convenios internacionales, Indret: Revista para el Análisis del Derecho, (4), 1-22. Con respecto a la contaminación del agua: El problemático asunto del derecho al agua y los acaparamiento de los recursos hídricos, ha sido objeto de atentos estudios por parte de los investigadores del Grupo Human Rights in Business (supra), en particular: Camarero Suárez, M. V. y Zamora Cabot, F. J. (2016) El acceso al agua limpia y al saneamiento: Un Derecho Humano crecientemente asediado. Huri - Age Consolider Ingenio. Papeles el Tiempo de los Derechos, (4). Recuperado de: https://redtiempodelosderechos. files.wordpress.com/2015/01/wp-4-16.pdf Sobre las obligaciones de las empresas y el derecho al agua, entre otros autores: i Solé, A. P. (2012). La situación en Colombia y la jurisdicción de la Corte Penal Internacional. Estudios de derecho internacional y derecho europeo en homenaje al profesor Manuel Pérez González , Vol. I (Tomo II), 939-968. Tirant lo Blanch; Fajardo del Castillo, T. (2012). Los bienes públicos del medio ambiente: el reto de la gestión sostenible de los recursos naturales en la Unión Europea. Anuario de la Facultad de Derecho de la Universidad Autónoma de Madrid, Carlos Espósito y Francisco José Garcimartín Alférez (coord.), (16), 219-246. Véase también:European Environment Agency. (2012) European waters - assessment of status and pressures. Recuperado de: http://www.eea.europa.eu/publications/european-waters-assessment-2012 Con respecto a la contaminación del suelo: Agencia europea de Medio Ambiente (2016) Uso del suelo. Recuperado de: http://www.eea.europa.eu/es/themes/landuse/intro

${ }^{7}$ Más información sobre los mapas elaborados por la OMS relativos a las estimaciones nacionales sobre la exposición a la contaminación del aire y sus repercusiones para la salud disponible en: http://www.who.int/mediacentre/news/releases/2016/airpollution-estimates/es/

8 "El medio ambiente en Europa: Estado y perspectivas 2015". Informe de síntesis, texto en: http://www.eea.europa.eu/soer-2015/ synthesis/el-medio-ambiente-en-europa. 
por ejemplo, de calidad del aire en muchas ciudades la contaminación sigue estando por encima de los niveles requeridos.

Según indica el Departamento de Salud Pública, Medio Ambiente y Determinantes Sociales de la Salud de la OMS: "Es sumamente urgente invertir en estrategias que reduzcan los riesgos ambientales en las ciudades, hogares y lugares de trabajo [...] Esas inversiones pueden reducir considerablemente la creciente carga mundial de enfermedades cardiovasculares y neumopatías, lesiones y cánceres y comportar una reducción inmediata en los costos sanitarios" ${ }^{\prime \prime}$ De ahí que uno de los objetivos prioritarios sea, en esta época caracterizada por el calentamiento global ${ }^{10}$ y fuertes riesgos por la salud generados por la contaminación del agua y de aire, que las ciudades lleguen a ser más sostenibles e inteligentes. Esto se puede alcanzar a través de la puesta en práctica de políticas de diseño y planificación urbana eficaces: destacan, entre otras varias, la lucha contra la mala calidad del aire, los altos niveles de ruido, las emisiones de gases de efecto invernadero, la escasez del agua, y una adecuada gestión de los residuos.

Resulta ser una verdad incontrovertible que los desafíos planteados en la actualidad tienen que ser resueltos a escala mundial, es decir, dentro de un plan de iniciativas globales que sea acordes con la máxima de "Vivir bien, respetando los límites ecológicos de nuestro planeta", tal como consta en el VII Programa General de Acción de la Unión Europea, en materia de medio ambiente, $2013-2020^{11}$ Con estos acuerdos la Unión ha acordado reducir las emisiones de gases de efectos invernaderos, detener la pérdida de biodiversidad, alcanzar niveles de calidad de aire que no den riesgos para la salud, proteger el medio ambiente y fomentar una transición hacia una economía verde entre otros fines. Dentro de lo que constituye este marco de acción, los desarrollos sostenible y equitativo serán, sin duda, los objetivos más necesarios de lograr. Para ello se deberá continuar con los acuerdos adoptados en la Conferencia Río + 20, conocida también como la Cuarta Cumbre de la Tierra, organizada por las Naciones Unidas, sobre Desarrollo Sostenible, que tuvo lugar en 2012 y tuco como eje central la Defensa de los Derechos Humanos en materia de medio ambiente. Una apuesta firme a favor de las energías renovables y de las mejoras de la eficacia energética debe contribuir a controlar el aumento de la temperatura media del planeta, que se ha situado cerca del $0,85 \%$ respecto a los niveles preindustriales, como se indica en el Quinto Informe del Panel Intergubernamental sobre

\footnotetext{
${ }^{9}$ Organización Mundial de la Salud. (2016) Cada año mueren 12,6 millones de personas a causa de la insalubridad del medio ambiente. Comunicado de prensa. Recuperado de: http://www.who.int/mediacentre/news/releases/2016/deaths-attributable-to-unhealthyenvironments/es/

${ }^{10}$ Sobre este aspecto hacemos referencia al Informe de la Agencia Europea del Medio Ambiente: "Vivir en un clima cambiante", donde se evidencia los efectos nocivos del cambio climático que puede agravar la contaminación atmosférica y las enfermedades respiratorias y cardiovasculares y conllevar a la muerte. Este informe se puede consultar en: http://www.eea.europa.eu/es/ publications/senales-de-la-aema-2015.

${ }^{11}$ Véase: Parlamento Europeo y el Consejo de la Unión Europea (2013) DECISIÓN № 1386/2013/UE. Recuperado de: http://eur-lex. europa.eu/legal-content/ES/TXT/?uri=CELEX\%3A32013D1386. El texto del Programa se puede consultar en: http://ec.europa.eu/ environment/pubs/pdf/factsheets/7eap/es.pdf. Véase también, Sánchez, D. F. D. G. (2013). El séptimo programa ambiental de la Unión Europea, 2013-2020. REVISTA ARAGONESA, (41-42), 71-121
} 
Cambio Climático ${ }^{12}$, base para los acuerdos de lucha contra sus efectos. En su memoria de 2016 quedó determinado que el calentamiento de las zonas tropicales y el Ártico se inició a partir de 1830. La transición energética y adaptación a los nuevos retos que se plantean en la esfera medioambiental tienen que dar paso, inexorablemente, a una sociedad más sostenible y baja en consumo de carbono, que se adapte a la problemática que representa el cambio climático.

El objeto de nuestro estudio constituye un tema de interés para todos aquellos que les inquieten los problemas actuales que surgen de las actividades empresariales en ámbito mundial, haciendo particular referencia al sector de los alimentos transgénicos. En él pretendemos destacar la encrucijada de los alimentos transgénicos en Estados Unidos, Europa y la OMC y las implicaciones sobre la protección del medio ambiente y la salud de las personas.

\section{CONEXIÓN EXISTENTE ENTRE EL DERECHO A UN AMBIENTE SANO Y LAS ACTIVIDADES DE LAS EMPRESAS}

La conexión de la problemática existente entre el derecho a un ambiente sano y las actividades de las empresas y su impacto sobre la salud de las personas ha quedado bien reflejada, a través de la Carta Encíclica Laudato Si, del Santo Padre Francisco, con fecha de 24 de mayo de $2015^{13}$, en los profundos razonamientos que exponemos, sucintamente, a continuación. Se refleja en su texto que la casa común, nuestra madre tierra, está sufriendo un deterioro ambiental global respecto de su suelo, agua, aire, seres vivientes, etc. Ocho años después de la famosa encíclica Pacem in Terris, el Santo Padre Pablo VI ya recogió la crisis ecológica consecuencia de la explotación inconsiderada de la naturaleza, bajo los efectos de la explosión de la civilización industrial. Su idea principal fue que el cambio climático es un problema global con graves dimensiones ambientales, sociales, económicas, distributivas y políticas. Lógicamente ha quedado demostrado que la mayor parte del calentamiento global de estas últimas décadas se debe a la gran concentración de gases de efecto invernadero emitidos por las actividades humanas y en particular a las actividades industriales y a la creciente contaminación ambiental ligadas al crecimiento económico. Asimismo, en su discurso ante la Organización de las Naciones Unidas para la Alimentación y la Agricultura (FAO) dejó sentado que los avances científico-técnicos y el crecimiento económico, de nada sirven si no se conjugan con el auténtico progreso, que no es otro que el social y el moral. De ahí que cada sistema jurídico tenga como reto incorporar la nueva visión del desarrollo sostenible, que es la misma visión que está impulsando la nueva agenda de las Naciones Unidas para el $2030^{14}$ sobre desarrollo

\footnotetext{
${ }^{12}$ Más información sobre el Grupo Intergubernamental de Expertos sobre el Cambio Climático (IPCC): http://www.ipcc.ch/home_ languages_main_spanish.shtml y sobre el Quinto Informe de Evaluación: https://www.ipcc.ch/report/ar5/index_es.shtml.

${ }^{13}$ Sobre este tema véanse también los estudios de Camarero Suarez, M.V. y Zamora Cabot, F. J. (2016)

${ }^{14}$ Con la Resolución de la Asamblea General del 25 de septiembre de 2015 se dio inicio a la agenda Transformar nuestro mundo: la Agenda 2030 para el Desarrollo Sostenible: Cumbre Histórica de las Naciones Unidas. (2015) La Agenda de Desarrollo Sostenible. Recuperado de: http://www.un.org/sustainabledevelopment/es/la-agenda-de-desarrollo-sostenible/ Oficialmente la agenda entró en vigor el 1 de enero de 2016. La información sobre los 17 nuevos objetivos de desarrollo sostenible en: Naciones Unidas. (2016) 17 objetivos para transformar nuestro mundo. Recuperado de: http://www.un.org/sustainabledevelopment/es/
} 
sostenible, cuyo propósito es crear sociedades que garanticen la plena satisfacción de las personas.

En consecuencia, llegamos a la firme conclusión de que el mundo actual en el que estamos inmersos requiere una drástica y urgente conversión ecológica global ante los retos que representa el cambio climático con su impacto negativo, especialmente, sobre los países en desarrollo. Este lema fue recogido con acierto en la ceremonia de apertura de los Juegos Olímpicos de 2016, celebrados en Río de Janeiro, a través de la imagen de un niño que, entre sus manos, entrelazaba una planta como símbolo de un compromiso por la defensa de la naturaleza. Nadie puede negar que nuestras futuras generaciones claman por un cambio radical de las políticas que están conduciendo a la creciente e imparable degradación ambiental, a una desprotección de la seguridad alimentaria y, con ello, al derecho a la salud de las personas. Además, se sigue incrementando día a día la íntima relación entre los desfavorecidos y la fragilidad del planeta, que debiera ser un bien común de todos.

\section{LA ENCRUCIJADA DE LOS ALIMENTOS TRANSGÉNICOS: ESTADOS UNIDOS, EUROPA Y LA OMC}

De esta manera, como botón de muestra, mencionamos otro de los grandes problemas a los que vamos a enfrentarnos ineludiblemente en un futuro inmediato, como es el relativo a los alimentos transgénicos. Así, podemos comprobar que la multinacional estadounidense Monsanto (una de las empresas con peor imagen pública del mundo) ha ido extendiendo paso a paso sus largos y poderosos tentáculos en cuanto a su capacidad de producción de alimentos genéticamente modificados. Merced a la implantación de múltiples redes comerciales, esta multinacional ha logrado expandirse a lo largo de todo el mundo, lo que ha logrado con una enorme rapidez a través de América Latina, Corea, Emiratos Árabes Unidos, Sudáfrica, etc.

Esta compañía agroquímica aceptó, tras 115 años desde su fundación, finalmente, en el pasado mes de septiembre de 2016 , la oferta de compra realizada por la farmacéutica alemana Bayer, más fuerte que Monsanto en el negocio de pesticida ${ }^{15}$ No obstante, la empresa estadounidense tiene ampliamente reconocida su prevalencia en el negocio de las semillas. Así, se producirá una combinación entre el negocio de semillas de Monsanto con el de la protección de cultivos, por parte de Bayer. Se abre, sin duda, en nuestra opinión, una nueva era en la agricultura. El dilema será tratar de conseguir alimentar a 3.000 millones de personas más en el mundo, en el año 2050, de una forma sostenible con el medio ambiente. Sin embargo, la reciente ola de fusiones que está teniendo lugar en el mundo en este sector industrial ${ }^{16}$ parece indicar que reducirá,

\footnotetext{
${ }^{15}$ Oferta que se cifró en 58.000 millones de euros, con el anuncio oficial de la fusión formalizado antes de la apertura del mercado de Wall Street.

${ }^{16}$ Recientemente, hemos conocido la concentración anunciada por las compañías canadienses Potash y Agrium, con el claro objetivo de reducir costes en la producción y distribución de fertilizantes, así como la fusión proyectada entre las norteamericanas Dupont y Dow Chemical, con gran peso específico en productos fitosanitarios, herbicidas destinados a colza, cereales, remolacha, etc.
} 
significativamente, las opciones de futuro de los agricultores, quienes verán reducido su margen de acción para poder llegar a acordar precios en el mercado. En consecuencia, la empresa combinada resultante seguirá con sus consolidadas sedes de semillas en Saint Louis (Estados Unidos) y de pesticidas en Monhein (Alemania), respectivamente. En orden a conseguir convertirse en el buque insignia en materia de suministros agrícolas (división en control de la suiza Syngenta, cuya compra fue anunciada este mismo año por la compañía estatal ChemChina, China National Chemical Corporation) tendrá que hacer serias concesiones a los reguladores de la competencia para que se le permita llevar a buen puerto la adquisición, cuyo cierre de operaciones está previsto para finales de 2017. En el supuesto de que no saliera adelante este acuerdo, el mayor de la historia de una firma alemana, Bayer se comprometió a indemnizar a Monsanto con una cantidad aproximada de 1.800 millones de euros.

En los Estados Unidos corresponde a la Agencia de Alimentos y Medicamentos/Drogas (Food and Drug Administration) la seguridad y el control de los productos alimenticios y los medicamentos humanos y veterinario. Apenas hay que retrotraerse a fechas recientes (1994) para establecer una primera comercialización, cuando apareció a la venta un tomate (Flavor Savr) producido para un masivo consumo humano, a través de la modificación de un gen que servía para retrasar su maduración. Obviamente, para que se permitiera su aparición y puesta en el mercado contó con la aprobación de dicha Agencia. Cuatro años más tarde, se autorizó en Europa el maíz transgénico Mon 810 de Monsanto, por el Consejo de la Unión Europea, que es el organismo con capacidad de decisión sobre la conveniencia de su distribución. En la actualidad, la mentada compañía se ha convertido en el indiscutible líder mundial de la producción de agroquímicos y biotecnología destinados a la agricultura e ingeniería genética de semillas y producción de herbicidas. Todo ello a pesar de la ausencia de estudios serios que avalen que su producción no está exenta de causar daño alguno para la salud humana. Se constata, además, en su modus operandi, la existencia de una concentración de tierras productivas en manos de pocos; en otras palabras, la "progresiva desaparición de pequeños productores que, como consecuencia de la pérdida de sus tierras explotadas, se han visto obligados a retirarse de la producción directa" En conclusión, este tipo de políticas comerciales no parece que colabore precisamente a la consecución del desarrollo equitativo de las técnicas agrícolas sostenibles. En la Unión Europea, por el contrario, existe una mayor especialización en cuanto a la regulación y control de esta materia, ya que se han creado dos organismos diferentes de acción para la vigilancia: la Autoridad Europea ${ }^{17}$, a cargo de la seguridad alimentaria y la Agencia Europa que se ocupa de la evaluación de los medicamentos.

Los consumidores de todo el mundo reclaman con urgencia que se les facilite una información precisa sobre sus características especiales, así como los efectos concretos que reúnen estos alimentos. Se requiere, por lo tanto, invertir sin mayor dilación, más fondos con la finalidad de promover su investigación a nivel mundial. Todo consumidor

\footnotetext{
${ }^{17}$ Autoridad que, como responsable de la evaluación del riesgo, dispone de un plazo de 6 meses para realizarla. La Comisión Europea que asume la gestión de dicho riesgo, lo presenta al llamado Comité Permanente de la Cadena Alimentaria y de Sanidad Animal.
} 
de bienes o usuario de servicios, en cualquier parte del planeta que resida, tiene derecho a conocer (right to know) todos los aspectos que están relacionados con cada uno de los tres derechos fundamentales que le amparan, a saber:

a) Derecho a la información.

b) Derecho a la salud y a la seguridad.

c) Derecho a la protección del medio ambiente.

Conviene observar, por un lado, en lo que incumbe a cada uno de estos tres derechos, que la seguridad alimentaria es una cuestión de elección del consumidor pero para hacer un uso eficaz de este derecho se necesita estar bien informado. Del mismo modo, la defensa del medio ambiente requiere una serie de conocimientos en torno a su problemática, que solamente pueden obtenerse a través de una información exacta y fidedigna, a la que en la mayoría de países del mundo se accede con dificultad.

En el continente europeo se vienen haciendo significativos esfuerzos económicos en esta dirección. Cabe citar que, a través de la puesta en marcha del VI Programa-Marco de Investigación y Desarrollo Tecnológico, en el seno de la UE, se adjudicó un presupuesto general de 17.500 millones de euros a una acción que se convirtió en uno de los principales instrumentos de la política de investigación comunitaria y el estudio de la biotecnología y organismos modificados genéticamente. Se han producido también avances legislativos en la materia, merced a la promulgación de Reglamentos CE 1829-1830/2003, dimanantes del Parlamento Europeo y del Consejo, sobre alimentos modificados genéticamente, trazabilidad y etiquetado de los mismos ${ }^{18}$ Todo operador industrial puede presentar su solicitud, de acuerdo con los Reglamentos, para cualquier producto alimenticio que contenga $O M G$, en tanto que cumpla con las normas de carácter superior previstas al respecto, como es el caso de la Directiva 2001/18/CE, sobre la liberación intencional de organismos modificados genéticamente.

La guerra comercial sobre los transgénicos no ha hecho más que comenzar. Tres países, Estados Unidos (el mayor productor, con una superficie cercana a los 100 millones de hectáreas ya en el año 2010), Canadá y Argentina (que, con Brasil y China, concentran el $98 \%$ de la producción mundial), presentaron una queja ante la Organización Mundial del Comercio (OMC) acusando a la Unión Europea de cerrar sus mercados a dichos alimentos, poniendo barreras a su importación al practicar una política de proteccionismo. Todo parece indicar que el futuro de la presencia de este invento biotecnológico, realizado por las grandes empresas, apunta hacia una mayor flexibilización en cuanto al levantamiento de su prohibición en la UE. Las resoluciones de este conflicto, a nuestro juicio, pasan por la tolerancia en su consumo, como así se lo hizo indicar la propia organización a

\footnotetext{
${ }^{18}$ Se entiende por trazabilidad (trace ability) la habilidad de rastrear y recopilar información hacia atrás en una cadena agroalimentaria, esto es, desde su origen hasta que alcanza al consumidor final. La sensibilidad del consumidor ha llevado a que, junto con el etiquetado, ambas facetas se muestren estrechamente relacionadas en la práctica.
} 
Rusia recientemente. De hecho, su auge ha adquirido tal expansión que sus normas regulatorias alcanzan incluso al viñedo. Sus modalidades más relevantes pertenecen a los cultivos de maíz (que cuenta con un creciente desarrollo en la agricultura, al ser un cereal esencial para la alimentación de la humanidad, además usado para la obtención del algodón y, a partir de éste, de la glucosa y fructosa y a los cultivos de algodón, colza y soja (base de la mayoría de productos que ingerimos diariamente, que representa un $50 \%$ del total cultivada en el mundo) Es de máximo interés recoger el dato de que la soja transgénica, auténtico oro para Monsanto (como propietaria de la semilla), y exportada por los grandes productores y las empresas multinacionales desde América Latina.

Precisamente, uno de los objetivos loables del Programa de Inocuidad Alimentaria en el que trabaja la OMS no es otro que el de colaborar con las autoridades nacionales en la identificación de alimentos que deben someterse a evaluaciones de riesgos, incluyendo aquellos alimentos genéticamente modificados (GM) y, por añadidura, recomendar las evaluaciones correctas. Los beneficios de estos Organismos Modificados Genéticamente (OMG), también denominados OGM (del Inglés Organisms Genetically Modified), frente a las variedades tradicionales, están siendo ampliamente estudiados por la mentada Organización.

Al menos esta orientación expuesta, en lo que incumbe al modus operandi de las grandes compañías multinacionales, no refleja en modo alguno, nuestro auténtico desideratum de la ecología integral. Esto es, un sistema que conduce a la necesaria imbricación de la ecología ambiental, económica y social, tal y como se deduce del mencionado texto de la Carta Encíclica Laudato Si' (2015, Capítulo III). Al contrario, parece que se abre una puerta que lleva a la miseria de los pueblos. Ello obedece a que esta orientación desconoce o ignora el indeleble significado de otra de las facetas clave que presenta el mentado problema, el olvido de la llamada ecología humana. Dentro de la esfera de la ecología cultural debemos reproducir el peligro existente con la aparición de la visión consumista de los seres humanos alentada por los engranajes de la actual economía globalizada (por ejemplo, este fenómeno de masas se refleja en la forma en la que se está abocando a un ensimismamiento por la búsqueda de las ropas de vestir de marcas de renombre, perseguidas por todos los jóvenes del mundo al unísono, a través de reputados centros o franquicias comerciales) que tiende a homogeneizar las culturas y a debilitar su variedad como auténticos tesoros de la humanidad (Padre Francisco, 2015, Capítulo IV).

\section{CONCLUSIONES}

Como consecuencia de todo lo expuesto con anterioridad, podemos llegar a establecer las siguientes conclusiones, a saber:

A nuestro juicio es indispensable un replanteamiento en el tema de la defensa de los derechos de la persona, plasmados en nuestro trabajo básicamente en cuanto a su vida y 
salud, junto a la protección del medio ambiente. Solamente una buena política ambiental en el marco de las empresas contribuirá a alcanzar el objetivo de proteger la salud de las personas y velar por la defensa del medio ambiente. Invertir en eco-innovación y nuevas tecnologías, medir el impacto ecológico de sus actividades y transmitir a inversores y clientes información medioambiental en sus informes anuales son las soluciones que se deben adoptar.

De esta forma afirmamos que la tecnología no puede estar ligada exclusivamente con las finanzas. Lo cierto es que el sistema mundial en el que estamos inmersos es insostenible al haber dejado de lado los fines que se relacionan con la acción humana. Un ejemplo muy significativo son los expuestos en relación a los alimentos transgénicos. Es aconsejable la toma de conciencia sobre la idea de que el progreso de la ciencia y de la técnica no equivale al avance del género humano y de la historia. La visión consumista del ser humano alentada por los engranajes de la actual economía debilita la inmensa variedad cultural de la humanidad. Necesitamos, en suma, cambiar el modelo de desarrollo global vigente hacia una aplicación más positiva y justa del mismo, ya que, de otro modo, corremos el enorme peligro de convertirnos en "gigantes de la técnica" y, al mismo tiempo, en "enanos del humanismo".

\section{REFERENCIAS BIBLIOGRÁFICAS}

Agencia europea de Medio Ambiente. (2015) El medio ambiente en Europa: Estado y perspectivas 2015 - Informe de síntesis. Recuperado de: http://www.eea.europa. eu/soer-2015/synthesis/el-medio-ambiente-en-europa

. (2015) Señales de la AEMA 2015 - Vivir en un clima cambiante. Recuperado de: http://www.eea.europa.eu/es/publications/senales-de-la-aema-2015

. (2016) Uso del suelo. Recuperado de: http://www.eea.europa.eu/es/themes/ landuse/intro

BBC MUNDO (2016) El mapa que muestra cuán contaminado está el aire que respiramos. Recuperado de: http://www.bbc.com/mundo/noticias-37501943

Camarero Suárez, M. V. y Zamora Cabot, F. J. (2016) El acceso al agua limpia y al saneamiento: Un Derecho Humano crecientemente asediado. Huri - Age Consolider Ingenio. Papeles el Tiempo de los Derechos, (4). Recuperado de: https:// redtiempodelosderechos.files.wordpress.com/2015/01/wp-4-16.pdf

Comisión Europea. (2013). Vivir bien, respetando los límites de nuestro planeta. VII PMA Programa General de Acción de la Unión en materia de Medio Ambiente hasta 2020. Recuperado de: http://ec.europa.eu/environment/pubs/pdf/factsheets/7eap/es.pdf 
Cumbre Histórica de las Naciones Unidas. (2015) La Agenda de Desarrollo Sostenible. Recuperado de: http://www.un.org/sustainabledevelopment/es/la-agenda-dedesarrollo-sostenible/

European Environment Agency. (2012) European waters - assessment of status and pressures. Recuperado de: http://www.eea.europa.eu/publications/europeanwaters-assessment-2012

Fajardo del Castillo, T. (2012). Los bienes públicos del medio ambiente: el reto de la gestión sostenible de los recursos naturales en la Unión Europea. Anuario de la Facultad de Derecho de la Universidad Autónoma de Madrid, Carlos Espósito y Francisco José Garcimartín Alférez (coord.), (16), 219-246.

Frisch, W (2015) Derecho penal y protección del clima: Posibilidades y límites de una contribución del Derecho penal a la protección del clima conforme a convenios internacionales, Indret: Revista para el Análisis del Derecho, (4), 1-22.

Grupo Intergubernamental de Expertos sobre el Cambio Climático. (2014) Quinto Informe de Evaluación. Recuperado de: http://www.ipcc.ch/home_languages_ main_spanish.shtml

iSolé, A. P. (2012). La situación en Colombia y la jurisdicción de la Corte Penal Internacional. Estudios de derecho internacional y derecho europeo en homenaje al profesor Manuel Pérez González , Vol. I (Tomo II), 939-968. Tirant lo Blanch.

Naciones Unidas. (2016) 17 objetivos para transformar nuestro mundo. Recuperado de: http://www.un.org/sustainabledevelopment/es/

Parlamento Europeo y el Consejo de la Unión Europea. (2001) Directiva 2001/18/CE. Recuperado de: http://www.wipo.int/wipolex/es/details.jsp?id=10335

. (2003) REGLAMENTO (CE) No 1830/2003. Recuperado de: http://eur-lex.europa.eu/ LexUriServ/LexUriServ.do?uri=OJ:L:2003:268:0024:0028:ES:PDF

. (2013) DECISIÓN N 1386/2013/UE. Recuperado de: http://eur-lex.europa.eu/legalcontent/ES/TXT/?uri=CELEX\%3A32013D1386

Organización Mundial de la Salud. (2016) Cada año mueren 12,6 millones de personas a causa de la insalubridad del medio ambiente. Comunicado de prensa. Recuperado de: http://www.who.int/mediacentre/news/releases/2016/deaths-attributableto-unhealthy-environments/es/ 
. (2016) Health and sustainable development. Recuperado de: http://www.who.int/ sustainable-development/news-events/breath-life/en/

. (2016) La OMS publica estimaciones nacionales sobre la exposición a la contaminación del aire y sus repercusiones para la salud. Recuperado de: http://www.who.int/ mediacentre/news/releases/2016/air-pollution-estimates/es/

Prüss-Ustün, A, et. al (2016) Preventing disease through healthy environments: A global assessment of the burden of disease from environmental risks. World Health Organization. Recuperado de: http://apps.who.int/iris/ bitstream/10665/204585/1/9789241565196_eng.pdf?ua=1

Sánchez, D. F. D. G. (2013). El séptimo programa ambiental de la Unión Europea, 20132020. REVISTA ARAGONESA, (41-42), 71-121.

Sí del Santo, C. E. L. Padre Francisco.(2015). Sobre el cuidado de la casa común. Bogotá: Paulinas.

The World Bank (2016) The cost of air pollution : strengthening the economic case for action (English) Recuperado de: http://documents.worldbank.org/curated/ en/781521473177013155/The-cost-of-air-pollution-strengthening-the-economiccase-for-action

XXIII, J. (1976). Carta Encíclica "Pacem in terris", 1963. Ocho Grandes Mensajes", BAC, Madrid, 225-229. 